\title{
BJP Vs Opposition Parties on Twitter: Social Media Strategies for Political Mobilisation During 2018 Karnataka and Telangana Assembly Elections
}

\author{
Sanjeev Ratna Singh
}

\begin{abstract}
BJP's 2014 Lok Sabha win under Narendra Modi was a watershed moment for politicians and social media users alike as it would change the very nature of political dissemination and its consumption. The extensive use of platforms like Twitter, Facebook etc. is reported to have played a crucial role in shaping the minds of first-time voters in favour of BJP. The post-2014 scenario in the country witnessed all main political parties embracing social media and gave it equal importance as compared to traditional tools of communication like newspapers, radio and TV. Twitter has now become one of the favourite tools of politicians for disseminating and interacting with social media users and creating a favourable impression among new and young voters.
\end{abstract}

Though BJP is still the biggest political force on social media in India, the change in digital strategies of other political parties like Congress and Rahul Gandhi also reflected in their rising Twitter engagement levels since 2017. Congress and other regional parties had to rework their strategy and take on BJP's aggressive political campaigns on digital platforms. The study is aimed to document how some regional parties and prominent leaders in south India transformed themselves as an attractive voting proposition among the electorate during the 2018 assembly elections.

This paper analyses comparative Twitter data of BJP, Congress, their top state leadership in Karnataka and Telangana along with leaders of regional parties like HD Kumaraswamy of the Janata Dal (Secular) and KT Rama Rao of the Telangana Rashtriya Samithi (TRS) during the 2018 Karnataka and Telangana assembly elections. It also includes an exhaustive analysis of official Twitter handles of Prime Minister Narendra Modi and Congress leader Rahul Gandhi on metrics like number of tweets, likes, replies with overall volume and engagement during the election campaigns. The paper aims to present a new understanding of how Twitter is being used as a tool for communication by political parties.

Keywords : Twitter, political communication, data mining, Congress, BJP, Telangana Rashtriya Samithi.

\section{INTRODUCTION}

Narendra Modi can be credited with inculcating digital discipline and acceptance in Indian politics post-2014. His adept usage of social media forced opposition parties to realise their importance forcing them quickly rework their

Revised Manuscript Received on September 15, 2019.

* Correspondence Author

Sanjeev Ratna Singh, P.hD. Scholar, GD Goenka University, Gurugram strategy. Narendra Modi remained the tallest leader on social media, especially Twitter in terms of volume and engagement and it was only a few years later that he started facing competition. The 2017 Gujarat assembly elections are an important milestone in this direction as this was the first time any non-BJP leader - Rahul Gandhi - managed to get close to Narendra Modi in terms of engagement on Twitter which has been documented by me elsewhere .

The other challenge before Narendra Modi was to replicate the social media strategy to win the popular vote in southern states where local languages and regional sentiment play an important role. Karnataka's politics is dominated by three parties: BJP, Congress and Janata Dal (Secular) and both national parties had allied with former Prime Minister HD Deve Gowda's, Janata Dal (Secular) to keep the other party out of power in the state over the past two decades. Congress had come to power in 2013 and the months preceding the Karnataka assembly elections saw the then sitting Congress chief minister, Siddaramaiah relied heavily on the Lingayat issue and Kannada pride against the so-called imperialist Hindi forces of BJP and the duo of Narendra Modi and BJP president Amit Shah.

The Telangana assembly election was different in many ways from Karnataka as this was one of the few states where BJP was not the dominant political party. Here, Telangana Rashtriya Samithi (TRS) and Congress fight for top honours followed by the likes of Telugu Desam Party (TDP), All India Majlis-e-Ittehadul Muslimeen (AIMIM) and BJP. Telangana chief minister $\mathrm{K}$ Chandrasekhar Rao had dissolved the assembly and called for early elections which led to Congress and Telugu Desam Party reaching a pre-poll alliance to counter any advantage for the ruling Telangana Rashtriya Samithi. K Chandrasekhar Rao did not have an official Twitter account as chief of Telangana Rashtriya Samithi but his party had an official account and most of the social media campaign including Twitter was handled by his son and senior minister KT Rama Rao .

This paper analyses the different Twitter strategies of BJP, Congress, their top leaders like Narendra Modi, Rahul Gandhi, Siddaramaiah, BS Yeddiyurappa and Janata Dal (Secular) leader HD Kumaraswamy in Karnataka. It also does a comparative analysis of BJP, Congress, Telangana Rashtriya Samithi and their top leadership including KT Rama

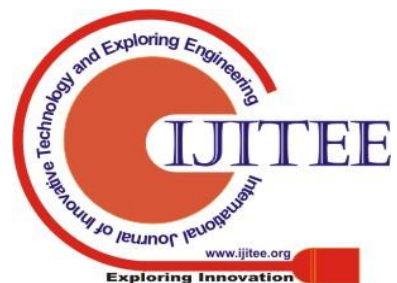


Rao during the 2018 assembly election campaign. The paper documents major issues highlighted by each party and their top leadership and their respective engagement on Twitter. The paper measures the engagement on metrics like tweets, retweets, likes, and replies on Twitter. The paper will help provide better insight into how political parties use Twitter as a tool for communication during election campaigns.

\section{METHODOLOGY}

This paper analyses the Twitter strategy of the official BJP, Congress, Narendra Modi and Rahul Gandhi handles and their top state leaders including former chief ministers Siddaramaiah, HD Kumaraswamy and current chief minister BS Yeddiyurappa for a six-month period between December 2017 - May 2018 in Karnataka. The paper also does a comparative analysis of Tweets posted by official Telangana BJP, Congress, All India Majlis-e-Ittehadul Muslimeen (AIMIM), and Telangana Rashtriya Samithi handles during the Telangana assembly election campaign between July December 2018. It also studies Twitter data for Telangana senior minister KT Rama Rao, state BJP president Dr K Laxman, state Congress chief Uttam Kumar Reddy and AIMIM chief, Asaduddin Owaisi. It provides an analysis of how these political parties and their leaders handled their election campaign and the major issues they chose to disseminate among their supporters. The paper analyses four major issues mentioned on Twitter: jobs, corruption, development and farmers. The data looks at Twitter engagement of their tweets, retweets, likes, and replies on the social media platform. This study aims to give a better perspective on Twitter usage for political messaging between national and regional parties and their leaders.

\section{INDIA'S DIGITAL MEDIA LANDSCAPE: A BACKGROUND}

India has undergone a sea change in the telecommunications with an increase in internet penetration and a sharp decrease in data prices. India has one of the cheapest mobile data in the world with an average of Rs 18.5 (USD 0.26) for $1 \mathrm{~GB}$ as per Cable.co.uk (a price comparison site $)^{\mathrm{i}}$. The number of smartphone users crossed the 400 million mark and the Kantar IMRB ICUBE report states India's internet user base is likely to touch 627 million by the end of $2019^{\mathrm{ii}}$. Twitter is one of the main social media platforms used by politicians across the world. It has become the norm for every big political leader at the national and international levels to disseminate and interact with people through the medium of Twitter. India is seventh on the list of countries with the highest number of Twitter users at 7.75 million while the US tops the list with 48.65 million $^{\mathrm{iii}}$.

BJP Vs Congress and Opposition Leaders on Twitter: Key Findings for Karnataka Assembly Elections (December 2017 - May 2018)

The Karnataka election was billed as a contest between the popularity of Narendra Modi vs Congress leader and chief minister Siddaramaiah who tried to stoke regional sentiment in order to retain power. A look at the total volume shows the official Congress and BJP Twitter handles being the most prolific tweeters putting out over 4,000 tweets during the six-month period between December 2017 - May 2018.
Narendra Modi and BJP's chief ministerial candidate BS Yeddiyurappa posted far more tweets than Congress leaders Rahul Gandhi, Siddaramaiah and Janata Dal (Secular) leader HD Kumaraswamy. But it was Rahul Gandhi who was getting better engagement levels than any other top politician.

For each of his 337 tweets, Rahul Gandhi was getting an average of 41,387 retweets per tweet and 118,671 likes per tweet during the six-month period of the Karnataka election campaign. Narendra Modi posted 1,727 tweets and had an average of 16,100 retweets per tweet and 73,891 likes per tweet. Siddaramaiah had the highest traction among regional leaders with an average of 3,402 retweets per tweet and 9,760 likes per tweet for the 761 tweets posted from his official Twitter handle. The official main handles for BJP and Congress posted 4,274 and 4,265 tweets respectively. BJP had an average of 2,778 retweets per tweet and 9,890 likes per tweet while Congress had an average of 3,309 retweets per tweet and 8,009 likes per tweet. BS Yeddiyurappa posted an average of 723 retweets per tweet and 2,725 likes per tweet in comparison with HD Kumaraswamy who had an average of 363 retweets per tweet and 1,978 likes per tweet. Rahul Gandhi had a maximum average of 15,605 replies per tweet compared to Narendra Modi's average of 3,954 replies per tweet. Siddaramaiah got engagement levels of an average of 823 replies per tweet. BJP and Congress main handles had an average of 559 and 676 replies per tweet respectively while BS Yeddiyurappa and HD Kumaraswamy had an average of 164 and 265 replies per tweet respectively.

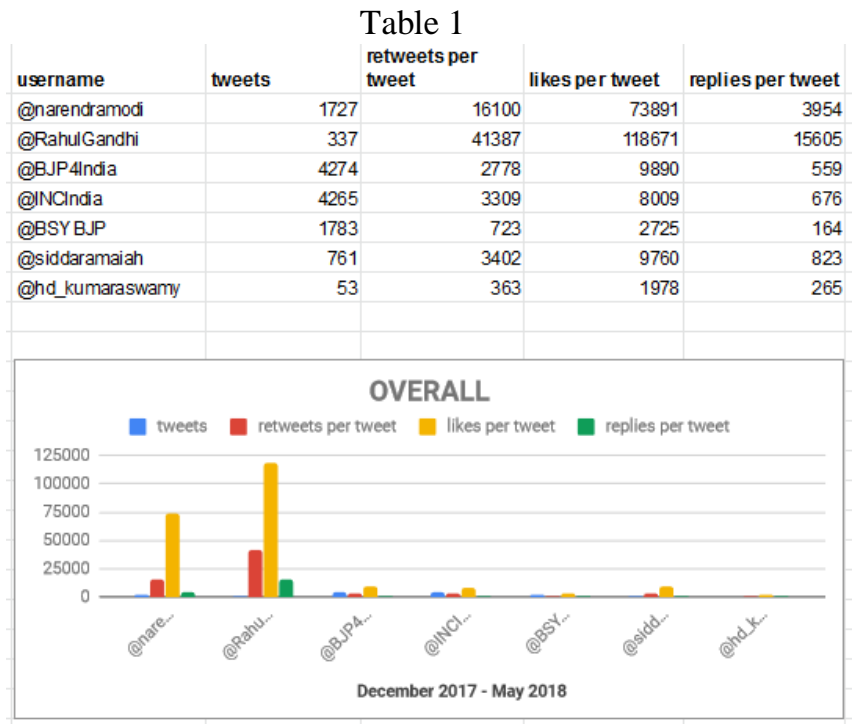

\section{BJP Vs Congress and Opposition Leaders on Jobs: Twitter comparison}

During this six-month period of the Karnataka election campaign, it was the main Congress handle that made the issue of jobs one of their prime focus. Congress leader Rahul Gandhi had the maximum engagement, his three tweets got an average of 12,654 retweets per tweet and 30,561 likes per tweet. Narendra Modi posted two tweets for an average of 3,782 retweets per tweet and 14,578 likes per tweet. Siddaramaiah tweeted 16 times on the issue of jobs and received an average of 1,000 retweets 
per tweet and 2,341 likes per tweet. BJP and Congress main handles put out 50 and 111 tweets related to the issue of jobs and got an average of 892 retweets per tweet, 2,810 likes per tweet and 1,009 retweets per tweet, 2,217 likes per tweet respectively. BS Yeddiyurappa posted 24 tweets getting an average engagement of 154 retweets per tweet and 610 likes per tweet while HD Kumaraswamy tweeted three times on the issue of jobs and got an average of 110 retweets per tweet and 464 likes per tweet. Rahul Gandhi also had the maximum average of 4,253 replies per tweet while Narendra Modi received an average of 559 replies per tweet. Siddaramaiah was the next leader to get an average of 162 replies per tweet. The official BJP and Congress Twitter handles received an average of 198 replies per tweet and 194 replies per tweet respectively. BS Yeddiyurappa and HD Kumaraswamy got an average of 41 replies per tweet and 51 replies per tweet respectively on the issue of jobs during the Karnataka election campaign.

Table 2

\begin{tabular}{|c|c|c|c|c|}
\hline username & tweets & retweets per tweet & likes per tweet & replies per tweet \\
\hline @narendramodi & 2 & 3782 & 14578 & 559 \\
\hline @RahulGandhi & 3 & 12654 & 30561 & 4253 \\
\hline @BJP4India & 50 & 892 & 2810 & 198 \\
\hline @INCIndia & 111 & 1009 & 2217 & 194 \\
\hline @BSYB.JP & 24 & 154 & 610 & 41 \\
\hline @siddaramaiah & 16 & 1000 & 2341 & 162 \\
\hline @hd_kumaraswamy & 3 & 110 & 464 & 51 \\
\hline
\end{tabular}

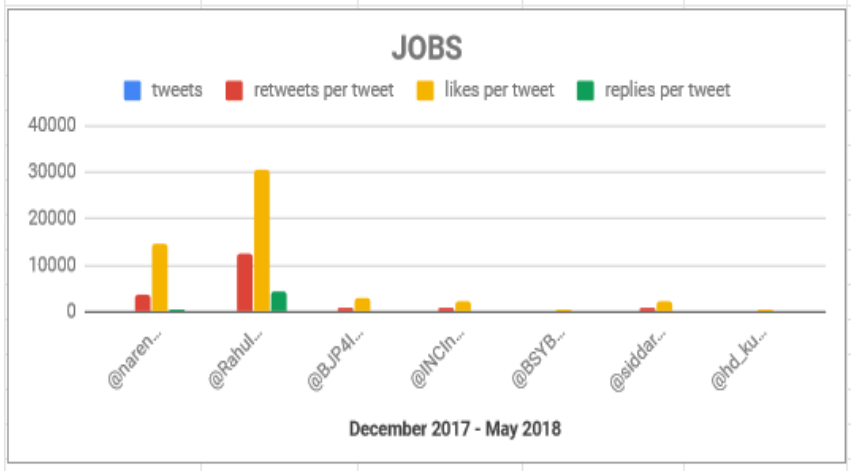

\section{BJP Vs Congress and Opposition Leaders on Corruption:} Twitter comparison

Overall, it was BJP leaders who were more vocal on the issue of corruption on Twitter during the election campaign, but Rahul Gandhi got the maximum traction on this issue as well. Rahul Gandhi received an average of 16,035 retweets per tweet and 38,782 likes per tweet for his four tweets on the issue of corruption. Narendra Modi posted 20 tweets and got an average of 5,432 retweets per tweet and 22,186 likes per tweet on the topic. Siddaramaiah put out 27 tweets while receiving an average engagement of 3,239 retweets per tweet and 7,299 likes per tweet. BJP and Congress main Twitter handles posted 68 and 80 corruption-related tweets respectively. BJP received an average of 886 retweets per tweet and 2,955 likes per tweet as compared to Congress which had an average of 1,018 retweets per tweet and 2,175 likes per tweet. BS Yeddiyurappa posted 62 tweets on the topic and got an average of 198 retweets and 605 likes per tweet. HD Kumaraswamy did not put out any corruption-related tweet during this six-month period between December 2017 - May 2018. Rahul Gandhi had the maximum traction on replies as well with an average of 4,968 replies per tweet followed by Narendra Modi with an average of 1,357 replies per tweet. Siddaramaiah received an average of 672 replies per tweet while BJP and Congress main handles got an average of 2018 and 201 replies per tweet. BS Yeddiyurappa had an average engagement of 48 replies per tweet. As mentioned above, HD Kumaraswamy did not post any corruption-related tweets during this period.

\begin{tabular}{|l|r|r|r|r|}
\hline \multicolumn{7}{c}{ Table 3 } & & \\
\hline username & tweets & \multicolumn{1}{c|}{ retweets per tweet } & likes per tweet & replies per tweet \\
\hline @narendramodi & 20 & 5432 & 22186 & 1357 \\
\hline @RahulGandhi & 4 & 16035 & 38782 & 4968 \\
\hline @BJP4India & 68 & 886 & 2955 & 208 \\
\hline @INCIndia & 80 & 1018 & 2175 & 201 \\
\hline @BSYBJP & 62 & 198 & 605 & 48 \\
\hline @Siddaramaiah & 27 & 3239 & 7299 & 672 \\
\hline @hd_kumaraswamy & 0 & 0 & 0 & 0 \\
\hline
\end{tabular}

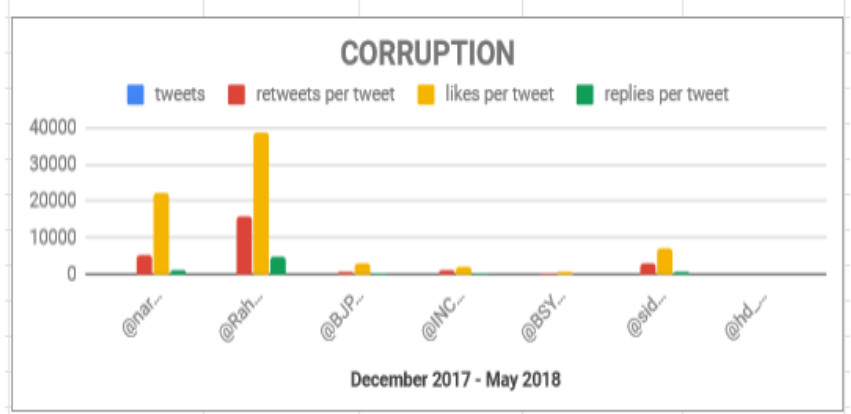

\section{BJP Vs Congress and Opposition Leaders on Farmers: Twitter comparison}

Both BJP and Congress tried to project the image of a farmer-friendly party on Twitter. Their main Twitter handles posted over 200 tweets on the topic during the six-month period of the Karnataka assembly election campaign. Rahul Gandhi had the maximum traction with an average of 12,959 retweets per tweet and 35,932 likes per tweet for his 11 tweets. Narendra Modi posted 69 tweets and received an average of 4,532 retweets per tweet and 20,048 likes per tweet. Siddaramaiah put out 51 farmer-related tweets and got an average of 1,256 retweets per tweet and 3,044 likes per tweet. BJP and Congress main handles posted 202 and 206 tweets on the issue of farmers. BJP had an average engagement of 704 retweets per tweet and 2,473 likes per tweet while Congress posted an average of 832 retweets and 1,892 likes per tweet. BS Yeddiyurappa posted 97 tweets related to farmers and received an average of 308 retweets per tweet and 1,022 likes per tweet while HD Kumaraswamy put out three tweets with an average of 83 retweets and 442 likes per tweet. Rahul had the maximum average of 4,815 replies per tweet followed by Narendra Modi and Siddaramaiah with an average of 1,106 and 216 replies per tweet. BJP and Congress handles received 155 and 129 replies per tweet respectively. BS Yeddiyurappa and HD Kumaraswamy got an average of 82 and 39 replies per tweet respectively. 
Table 4

\begin{tabular}{|l|r|r|r|r|}
\hline username & tweets & \multicolumn{1}{c|}{ retweets per tweet } & likes per tweet & replies per tweet \\
\hline @narendramodi & 69 & 4532 & 20048 & 1106 \\
\hline @RahulGandhi & 11 & 12959 & 35932 & 4815 \\
\hline @BJP4India & 202 & 704 & 2473 & 155 \\
\hline @NCIndia & 206 & 832 & 1892 & 129 \\
\hline @BSYBJP & 97 & 308 & 1022 & 82 \\
\hline @Siddaramaiah & 51 & 1256 & 3044 & 216 \\
\hline @hd_kumaraswamy & 3 & 83 & 442 & 39 \\
\hline
\end{tabular}

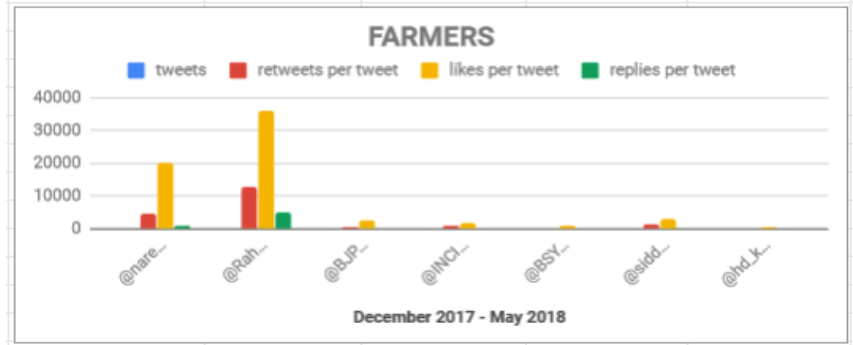

BJP Vs Congress and Opposition Leaders on Development: Twitter comparison

BJP has been aggressive on the issue of development and it reflected in their Twitter campaign strategy as well. The main BJP handle posted over 200 tweets while their leaders posted over 100 tweets on the issue. But Rahul Gandhi still had the maximum engagement with an average of 15,582 retweets per tweet and 40,600 likes per tweet for his three tweets on the issue of development. Narendra Modi posted 134 tweets getting an average engagement of 6,453 retweets per tweet and 28,228 likes per tweet followed by Siddaramaiah who had an average engagement of 1,283 retweets per tweet and 3,846 likes per tweet for his 32 tweets on the topic. The main BJP handle posted 291 tweets with an average engagement of 826 retweets per tweet and 3,054 out 92 tweets for an average of 884 retweets per tweet and 2,028 likes per tweet. BS Yeddiyurappa tweeted 173 times during the six-month period for an average engagement of 274 retweets per tweet and 1,033 likes per tweet. HD Kumaraswamy posted three tweets for an average of 98 retweets per tweet and 570 likes per tweet. Rahul Gandhi had the maximum average of 5,540 replies per tweet while Narendra Modi had an average of 1,822 replies per tweet followed by Siddaramaiah who received an average of 334 replies per tweet. BJP and Congress handles posted an average of 186 and 142 replies per tweet while BS Yeddiyurappa and HD Kumaraswamy got an average of 64 and 51 replies per tweet.

Table 5

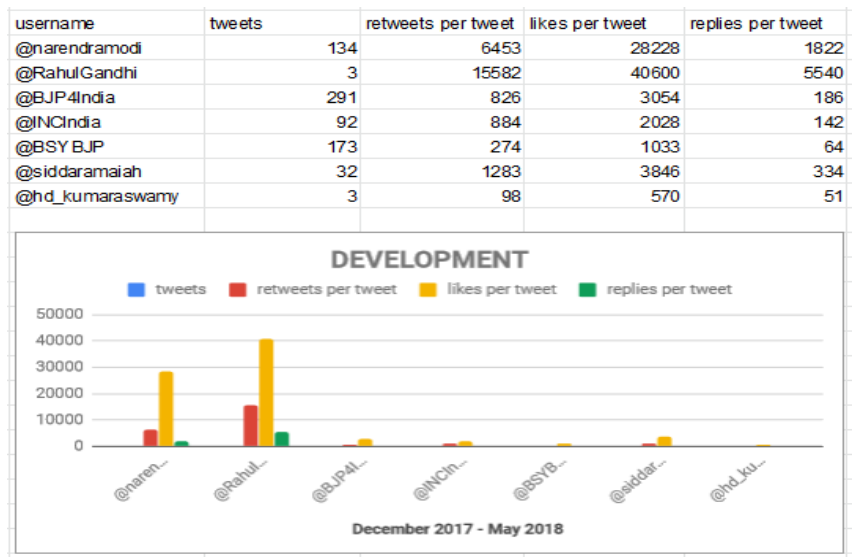
likes per tweet as compared to the Congress handle which put

BJP Vs Congress and Opposition Leaders on Karnataka: Twitter comparison

Rahul Gandhi received the maximum average engagement on Karnataka-related tweets during the six-month election campaign. The Congress leader posted 29 tweets and received an average of 11,403 retweets Congress leader and 32,500 likes per tweet on this issue. Narendra Modi tweeted 103 times on the topic and received an average engagement of 4,666 retweets per tweet and 20,045 likes per tweet followed by Siddaramaiah who posted 285 tweets for an average of 1,334 retweets per tweet and 3,717 likes per tweet. BJP main Twitter handle posted 510 tweets and received an average engagement of 847 retweets per tweet and 2,944 likes per tweet. The main Congress handle put out the maximum number of 818 tweets for an average of 791 retweets per tweet and 1,825 likes per tweet. BS Yeddiyurappa and HD Kumaraswamy posted 539 and 17 Karnataka-related tweets respectively. BS Yeddiyurappa had an average engagement of 327 retweets per tweet and 1,071 likes per tweet while HD Kumaraswamy received an average of 142 retweets and 739 likes per tweet. Rahul had the maximum average of 3,305 replies per tweet while Narendra Modi got an average of 1,191 replies per tweet with Siddaramaiah receiving an average of 319 replies per tweet. BJP and Congress got an average of 160 and 120 replies per tweet respectively. BS Yeddiyurappa and HD Kumaraswamy received an average engagement of 74 replies per tweet and 103 replies per tweet respectively.

\begin{tabular}{|c|c|c|c|c|}
\hline username & tweets & retweets per tweet & likes per tweet & replies per tweet \\
\hline @narendramodi & 103 & 4666 & 20045 & 1191 \\
\hline @RahulGandhi & 29 & 11403 & 32500 & 3305 \\
\hline @BJP4lndia & 510 & 847 & 2944 & 160 \\
\hline @INCIndia & 818 & 791 & 1825 & 120 \\
\hline @BSYBJP & 539 & 327 & 1071 & 74 \\
\hline @siddaramaiah & 285 & 1334 & 3717 & 319 \\
\hline @hd_kumaraswamy & 17 & 142 & 739 & 103 \\
\hline
\end{tabular}

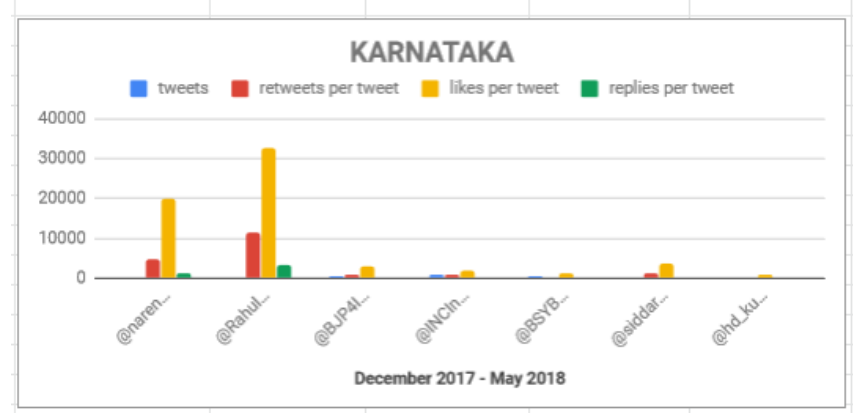

If one looks at overall figures and the volume, it is Narendra Modi who leads the pack with 4, 543,645 retweets and 20,723,254 likes for his 1,727 tweets during the six-month period from December 2017-May 2018. Rahul Gandhi posted 337 tweets and received overall figures of 2,185,408 retweets and 6,300,564 likes during the same period. The main BJP and Congress Twitter handles posted 4,274 and 4,265 tweets respectively. BJP handle received overall engagement of 1,916,198 retweets and 6,782,393 likes while 
Congress had a total of 2,250,969 retweets and 5,393,248 likes. Siddaramaiah had the best engagement among regional leaders, he posted 761 tweets and had a total of 463,007 retweets and 1,312,815 likes. BS Yeddiyurappa put out 1,783 tweets and received 208,807 retweets and 782,631 likes followed by HD Kumaraswamy who got overall figures of 3,883 retweets and 21,114 likes for his 53 tweets. Narendra Modi also had the maximum total of 1,105,996 replies while Rahul Gandhi had 812,859 replies. The BJP and Congress handles received 382,248 and 461,865 replies respectively. Siddaramaiah had overall 108,764 replies as compared to BS Yeddiyurappa and HD Kumaraswamy who received 46,522 and 3,316 replies respectively.

Table 7:

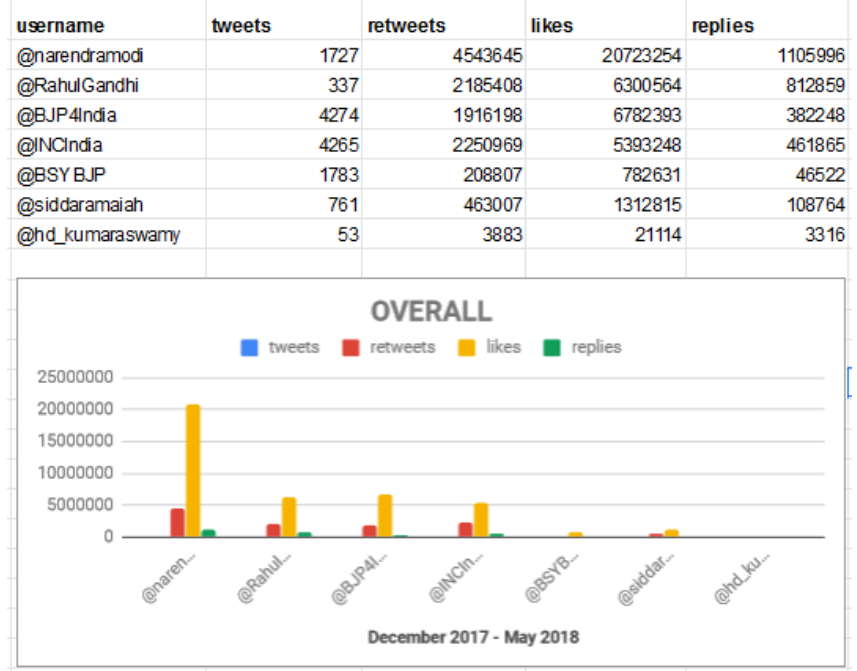

Telangana Rashtriya Samithi Vs Congress, BJP and Opposition Leaders on Twitter: Key Findings for Telangana Assembly Elections (July 2018 - December 2018)

The Telangana assembly elections witnessed a direct fight between the Telangana Rashtriya Samithi (TRS) and Congress which had entered an alliance with the Telugu Desam Party (TDP) while BJP remained a fringe player. Yet it was Narendra Modi who posted the maximum number of 2030 tweets during the six-month period between July December 2018 and received an average engagement 16,024 retweets per tweet and 73,383 likes per tweet. Rahul Gandhi tweeted 286 times for an average of 46,762 retweets per tweet and 158,083 likes per tweet. In terms of regional leaders, KT Rama Rao of TRS posted 969 tweets during the same period and received an average of 1,502 retweets per tweet and 13,111 likes per tweet. The Telangana BJP president Dr K Laxman posted 335 tweets getting an average of 10 retweets per tweet and 51 likes per tweet. The Telangana Congress president Uttam Kumar Reddy tweeted 190 times with an average engagement of 152 retweets per tweet and 653 likes per tweet. Asaduddin Owaisi of the All India Majlis-e-Ittehadul Muslimeen (AIMIM) tweeted 485 times and had an average of 1,529 retweets per tweet and 5,528 likes per tweet. The state Twitter handles of BJP, Congress and TRS posted 1,310,1,496 and 1,883 tweets respectively while AIMIM tweeted 151 times during this period. The state BJP handle had an average of 71 retweets per tweet and 225 likes per tweet while the state Congress handle received an average engagement of 304 retweets per tweet and 582 likes per tweet. TRS got an average of 172 retweets per tweet and 1,503 likes per tweet while AIMIM received an average of 50 retweets per tweet and 193 likes per tweet.

Rahul Gandhi had the maximum average of 17,728 replies per tweet while Narendra Modi received an average of 3,157 replies per tweet. KT Rama Rao had an average of 799 replies per tweet while Dr K Laxman and Uttar Kumar Reddy received an average of 237 and 2,309 replies per tweet respectively. Asaduddin Owaisi had an average of 328 replies per tweet. The state BJP and Congress handles got an average of 07 and 36 replies per tweet while TRS and AIMIM received an average of 64 and 07 replies per tweet.

\begin{tabular}{l|rrrrr} 
Table 8: & \multicolumn{1}{c}{} \\
\hline username & tweets & & retweets per tweet & likes per tweet & replies per tweet \\
\hline @narendramodi & 2030 & 16024 & 73383 & 3157 \\
\hline @RahulGandhi & 286 & 46762 & 158083 & 17728 \\
\hline @KTRTRS & 969 & 1502 & 13111 & 799 \\
\hline @drlaxmanbjp & 335 & 10 & 51 & 237 \\
\hline @UttamTPCC & 190 & 152 & 653 & 2309 \\
\hline @asadowaisi & 485 & 1529 & 5528 & 328 \\
\hline @BJP4Telangani & 1310 & 71 & 225 & 7 \\
\hline @INCTelangana & 1496 & 304 & 582 & 36 \\
\hline @trsparty online & 1883 & 172 & 1503 & 64 \\
\hline @aimim_nationa & 151 & 50 & 193 & 7 \\
\hline
\end{tabular}
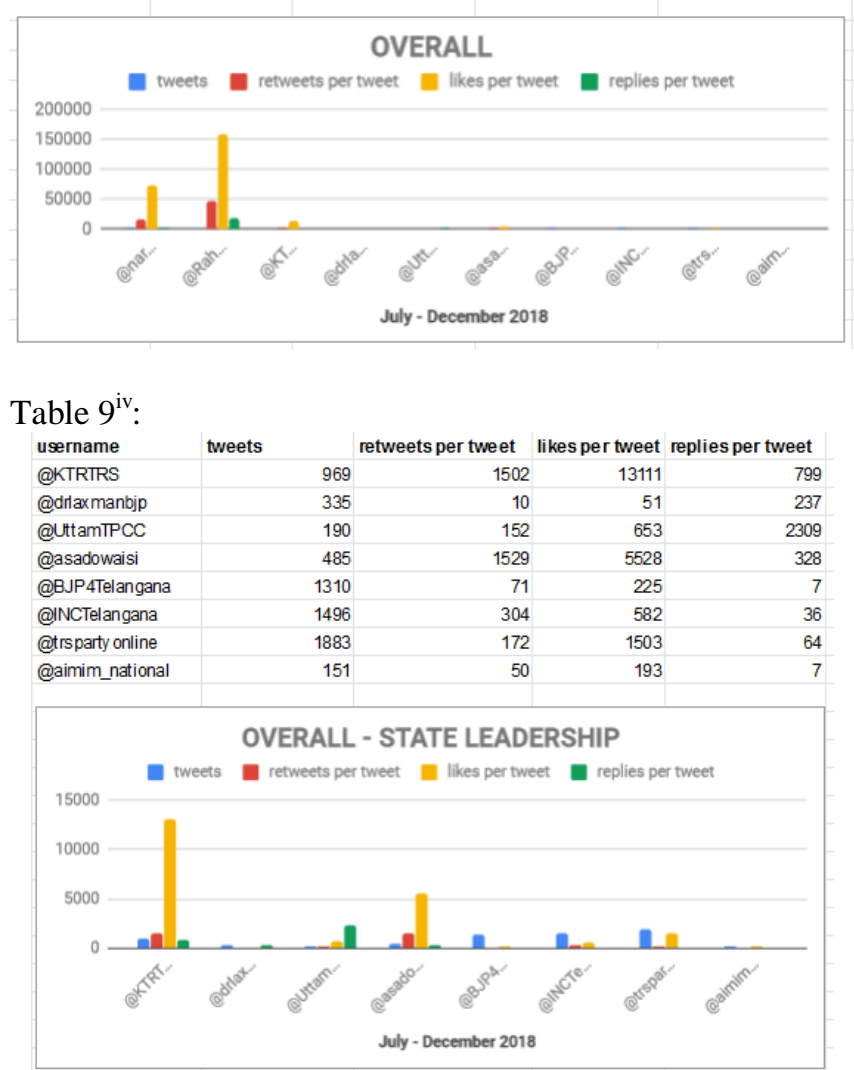

TRS Vs Congress and BJP on Jobs: Twitter comparison Rahul Gandhi had the maximum traction on the issue of jobs during the election campaign with his six tweets getting an average engagement of 15,281 retweets and 41,537 likes per tweet. Narendra Modi posted five job-related tweets and received an average of 4,980 retweets per tweet and 18,266 likes per tweet. KT Rama Rao had 12 tweets on the topic and he received an average engagement of 405 retweets and 3,507 likes per tweet. 
BJP's Dr K Laxman posted three tweets and had an average engagement of 12 retweets per tweet and 36 likes per tweet. Congress' Uttam Kumar Reddy received an average engagement of 51 retweets per tweet and 105 likes per tweet for his three tweets related to jobs. The state BJP handle put out 14 tweets and had an average of 16 retweets and 49 likes per tweet. It was the state Congress handle which posted the maximum number of 53 job-related tweets and had an average engagement of 106 retweets per tweet and 185 likes per tweet. The TRS official handle had seven related tweets with an average of 95 retweets per tweet and 741 likes per tweet. Rahul Gandhi also had the maximum average of 4,914 replies per tweet while Narendra Modi has an average of 889 replies per tweet. KT Rama Rao had an average of 201 replies per tweet. Dr K Laxman and Uttam Kumar Reddy had an average of one and eight replies per tweet on this topic. The state BJP and Congress handles had an average engagement of one and 12 replies per tweet on the issue of jobs during the six-month period. The TRS handle received an average of 91 replies per tweet.

Table $10^{\mathrm{v}}$ :

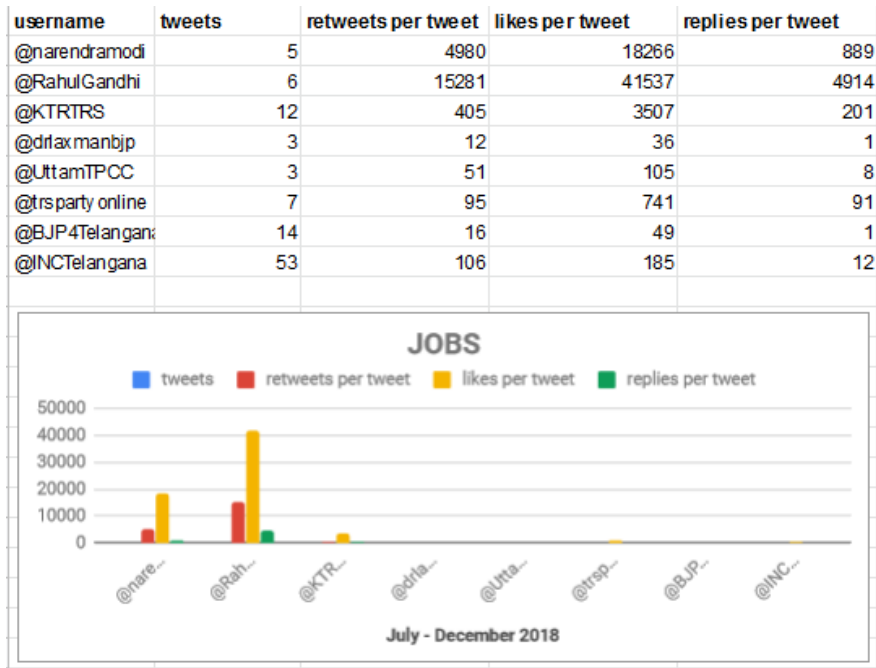

Table $11^{\text {vi }}$ :

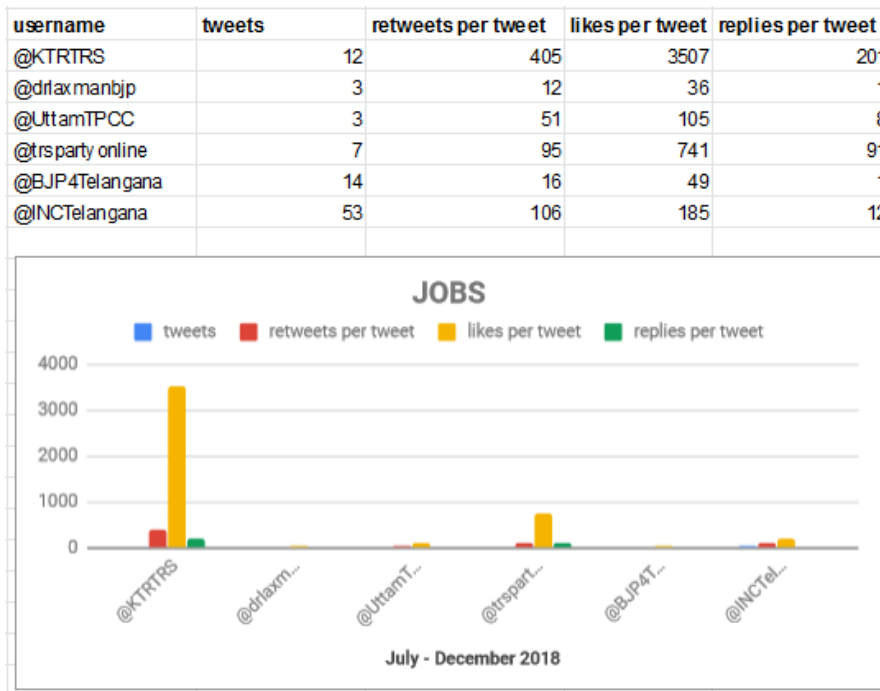

TRS Vs Congress and BJP on Corruption: Twitter comparison

Rahul Gandhi led the way in terms of engagement on the issue of corruption during this period with his nine tweets receiving an average of 14,023 retweets per tweet and 38,512 likes per tweet. Narendra Modi posted 15 corruption-related tweets and had an average of 7,529 retweets and 30,117 likes per tweet. KT Rama Rao had three tweets on the issue of corruption and he got an average of 2,591 retweets per tweet and 12,104 likes per tweet. Dr K Laxman posted only one tweet on corruption with nine retweets and 48 likes while Uttam Kumar Reddy tweeted eight times for an average engagement of 339 retweets per tweet and 918 likes per tweet. The state BJP handle posted 16 tweets on the topic and had an average of 19 retweets per tweet and 57 likes per tweet. The state Congress handle put out 23 tweets and received an average engagement of 103 retweets per tweet and 180 likes per tweet. The TRS handle had two tweets for an average of 33 retweets per tweet and 109 likes per tweet. Rahul Gandhi had the maximum average of 4,687 replies per tweet followed by Narendra Modi with an average of 2,170 replies per tweet. KT Rama Rao had an average of 1,140 replies per tweet. Dr K Laxman and Uttam Kumar Reddy had an average of one reply per tweet and 58 replies per tweet respectively. The state BJP and Congress handles had an average of two and 11 replies per tweet while the TRS handle had an average of two replies per tweet.

Table $12^{\text {vii. }}$

\begin{tabular}{|l|rrrrr|}
\hline username & tweets & \multicolumn{2}{c}{ retweets per tweet } & likes per tweet & \multicolumn{2}{c|}{ replies per tweet } \\
\hline @narendramodi & 15 & 7529 & 30117 & 2170 \\
\hline @RahulGandhi & 9 & 14023 & 38512 & 4687 \\
\hline @KTRTRS & 3 & 2591 & 12104 & 1140 \\
\hline @drlaxmanbjp & 1 & 9 & 48 & 1 \\
\hline @UttamTPCC & 8 & 339 & 918 & 58 \\
\hline @BJP4Telangani & 16 & 19 & 57 & 2 \\
\hline @NCTelangana & 23 & 103 & 180 & 11 \\
\hline @trsparty online & 2 & 33 & 109 & 2 \\
\hline & & & & &
\end{tabular}
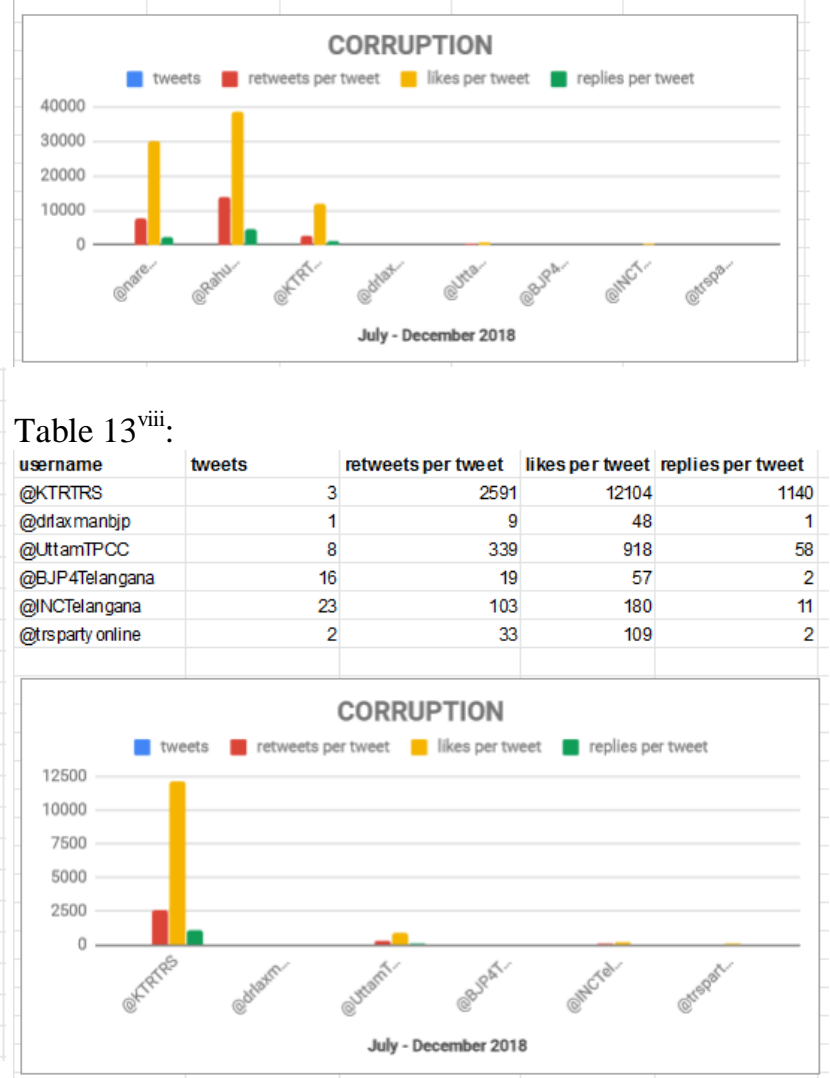
TRS Vs Congress and BJP on Farmers: Twitter comparison

Farmers have remained one of the top priorities for politicians and the Telangana assembly election too saw a fair share of tweets being devoted towards the issue of farmers and agriculture. Rahul Gandhi had 17 tweets on the issue and received an average engagement of 14,870 retweets per tweet and 52,218 likes per tweet. Narendra Modi posted 65 farmer-related tweets and got an average of 5,746 retweets per tweet and 24,262 likes per tweet while KT Rama Rao had 14 tweets with an average engagement of 433 retweets per tweet and 3,614 likes per tweet. BJP's Dr K Laxman posted five farmer-related tweets and received an average of 20 retweets per tweet and 88 likes per tweet while Uttam Kumar Reddy's six tweets on the subject got an average engagement of 618 retweets per tweet and 2,367 likes per tweet. The state BJP handle posted five tweets on the topic and received an average of 17 retweets and 36 likes while the state Congress handle posted 45 tweets and had an average engagement of 94 retweets and 170 likes per tweet. The TRS handle posted 29 farmer-related tweets and received an average engagement of 43 retweets per tweet and 339 likes per tweet. Rahul Gandhi and Narendra Modi received an average of 6,994 replies per tweet and 1,249 replies per tweet respectively. KT Rama Rao had an average of 250 replies per tweet. Dr K Laxman and Uttam Kumar Reddy received an average of three and 92 replies per tweet on the issue of farmers. The state BJP handle had an average of less than one reply per tweet while the state Congress handle received an average engagement of eight replies per tweet. The TRS handle had an average of 15 replies per tweet.

\begin{tabular}{|l|r|r|r|r|}
\hline Table $14^{\text {ix }}$ : & \multicolumn{3}{c|}{} \\
\hline username & tweets & \multicolumn{1}{c|}{ retweets per tweet } & likes per tweet & replies per tweet \\
\hline @narendramodi & 65 & 5746 & 24262 & 1249 \\
\hline @RahulGandhi & 17 & 14870 & 52218 & 6994 \\
\hline @KTRTRS & 14 & 433 & 3614 & 250 \\
\hline @drlaxmanbjp & 5 & 20 & 88 & 3 \\
\hline @UttamTPCC & 6 & 618 & 2367 & 92 \\
\hline @BJP4Telangan: & 5 & 17 & 36 & 0 \\
\hline @NCTelangana & 45 & 94 & 170 & 8 \\
\hline @trsparty online & 29 & 43 & 339 & 15 \\
\hline
\end{tabular}

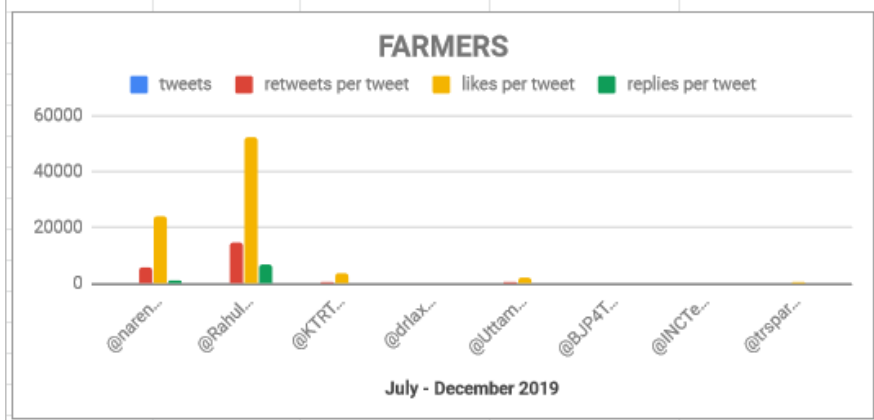

\begin{tabular}{|c|c|c|c|c|}
\hline username & tweets & retweets per tweet & likes per tweet & replies per tweet \\
\hline @KTRTRS & 14 & 433 & 3614 & 250 \\
\hline @driaxmanbjp & 5 & 20 & 88 & 3 \\
\hline @UttamTPCC & 6 & 618 & 2367 & 92 \\
\hline @BJP4Telangana & 5 & 17 & 36 & 0 \\
\hline @|NCTelangana & 45 & 94 & 170 & 8 \\
\hline @trsparty online & 29 & 43 & 339 & 15 \\
\hline
\end{tabular}

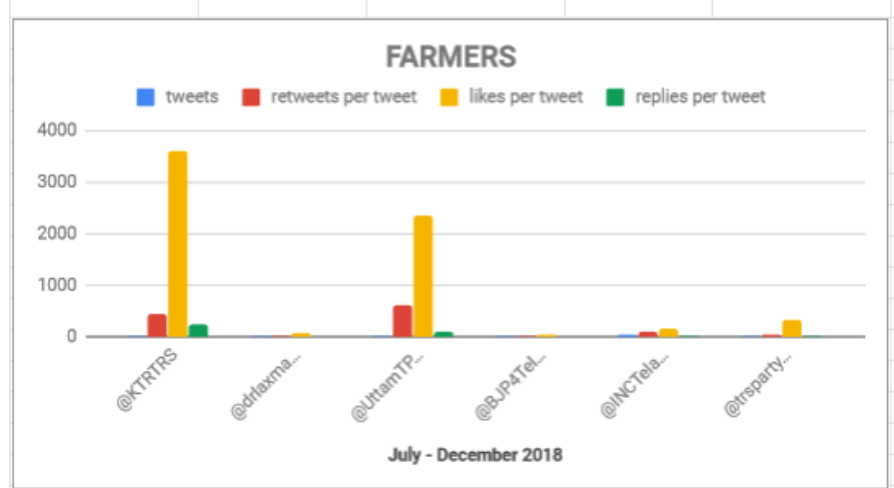

TRS Vs Congress and BJP on Development: Twitter comparison

Prime Minister Narendra Modi posted a maximum of 115 tweets related to development during this six-month period from July - December 2018 and received an average engagement of 4,729 retweets per tweet and 20,906 likes per tweet. Rahul Gandhi had only on development-related tweet for which he received 5,107 retweets and 15,539 likes. KT Rama Rao posted 16 tweets on the topic and received an average engagement of 514 retweets per tweet and 4,436 likes per tweet. BJP's state unit chief Dr K Laxman tweeted 14 times on development-related issues and got an average of 18 retweets per tweet and 65 likes per tweet. Uttam Kumar Reddy posted seven tweets and received an average engagement of 141 retweets per tweet and 434 likes per tweet. The state BJP handle put out 47 related tweets for an average of 21 retweets and 70 likes per tweet. The state Congress handle posted 18 tweets and received an average engagement of 98 retweets per tweet and 175 likes per tweet. The TRS handle had 105 tweets on development and received an average of 59 retweets per tweet and 473 likes per tweet. Rahul Gandhi had the maximum average of 1,134 replies per tweet while Narendra Modi received an average of 925 replies per tweet followed by KT Rama Rao who had an average of 260 replies per tweet. Dr K Laxman received an average of one reply per tweet while Uttam Kumar Reddy had an average of 41 replies per tweet. The state BJP and Congress handles received an average engagement of three and nine replies per tweet while the TRS handle got an average of 21 replies per tweet. 


\begin{tabular}{|c|c|c|c|c|}
\hline username & tweets & retweets per tweet & likes per tweet & replies per tweet \\
\hline @narendramodi & 115 & 4729 & 20906 & 925 \\
\hline @RahulGandhi & 1 & 5107 & 15539 & 1134 \\
\hline @KTRTRS & 16 & 514 & 4436 & 260 \\
\hline @drlaxmanbjp & 14 & 18 & 65 & 1 \\
\hline @UttamTPCC & 7 & 141 & 434 & 41 \\
\hline @BJP4Telangan: & 47 & 21 & 70 & 3 \\
\hline @INCTelangana & 18 & 98 & 175 & 9 \\
\hline @trsparty online & 105 & 59 & 473 & 21 \\
\hline
\end{tabular}

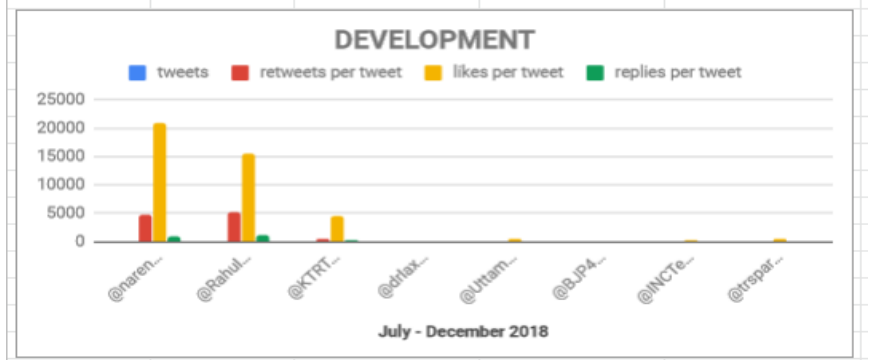

Table $17^{\mathrm{xii}}$ :

\begin{tabular}{|l|rrrrr|}
\hline username & tweets & retweets per tweet & likes per tweet & replies per tweet \\
\hline @KTRTRS & 16 & 514 & 4436 & 260 \\
\hline @drlaxmanbjp & 14 & 18 & 65 & 1 \\
\hline @UttamTCC & 7 & 141 & 434 & 41 \\
\hline @BJP4Telangana & 47 & 21 & 70 & 3 \\
\hline @INCTelangana & 18 & 98 & 175 & 9 \\
\hline @trsparty online & 105 & 59 & 473 & 21
\end{tabular}

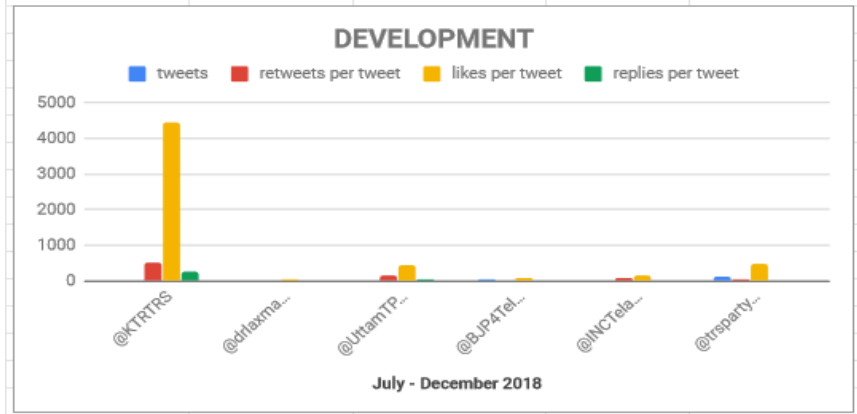

TRS Vs Congress and BJP on Telangana: Twitter comparison

The issue of Telangana pride seems to have been a major electoral issue as most parties and their leaders chose to talk more about the same on Twitter with BJP and TRS posting over a thousand tweets on the issue. Rahul Gandhi posted seven Telangana-related tweets and received an average of 9,544 retweets per tweet and 37,299 likes per tweet. Narendra Modi had 31 related tweets for an average engagement of 3,540 retweets per tweet and 17,004 likes per tweet. KT Rama Rao tweeted 201 times on the issue of Telangana and had an average of 681 retweets per tweet and 5,345 likes per tweet. Dr K Laxman posted 201 Telangana related tweets and received an average of 18 retweets per tweet and 82 likes per tweet. Uttam Kumar Reddy tweeted 101 times with an average engagement of 240 retweets per tweet and 849 likes per tweet. Asaduddin Owaisi posted 45 tweets for an average of 334 retweets per tweet and 1,471 likes per tweet. The state BJP handle put out 1,233 tweets with an average of 23 retweets per tweet and 72 likes per tweet. The state Congress handle posted 691 tweets with an average of 99 retweets per tweet and 1,471 likes per tweet. The TRS handle had 1,270 tweets with an average of 58 retweets per tweet and 457 likes per tweet.

The AIMIM handle posted six tweets and received an average engagement of 29 retweets per tweet and 145 likes per tweet. Rahul Gandhi had the maximum average of 2,543 replies per tweet followed by Narendra Modi and KT Rama Rao who received an average of 638 and 370 replies per tweet respectively. Dr Laxman and Uttam Kumar Reddy had an average of three and 59 replies per tweet respectively on this topic. Asaduddin Owaisi had an average of 79 replies per tweet. The state BJP and Congress handles received an average of one and 11 replies per tweet respectively. The TRS and AIMIM handles received an average engagement of 19 replies per tweet and six replies per tweet respectively.

\begin{tabular}{|c|c|c|c|c|}
\hline uærname & tweets & retweets per tweet & likes per tweet & replies per tweet \\
\hline @narendramodi & 31 & 3540 & 17004 & 638 \\
\hline @RahulGandhi & 7 & 9544 & 37299 & 2543 \\
\hline @KTRTRS & 201 & 681 & 5345 & 370 \\
\hline @drlaxmanbjp & 201 & 18 & 82 & 3 \\
\hline @UttamTPCC & 101 & 240 & 849 & 59 \\
\hline @asadowaisi & 45 & 334 & 1471 & 79 \\
\hline @BJJP4Telangan: & 1233 & 23 & 72 & 1 \\
\hline @INCTelangana & 691 & 99 & 193 & 11 \\
\hline @trsparty online & 1270 & 58 & 457 & 19 \\
\hline @aimim_nationa & 6 & 29 & 145 & 6 \\
\hline
\end{tabular}
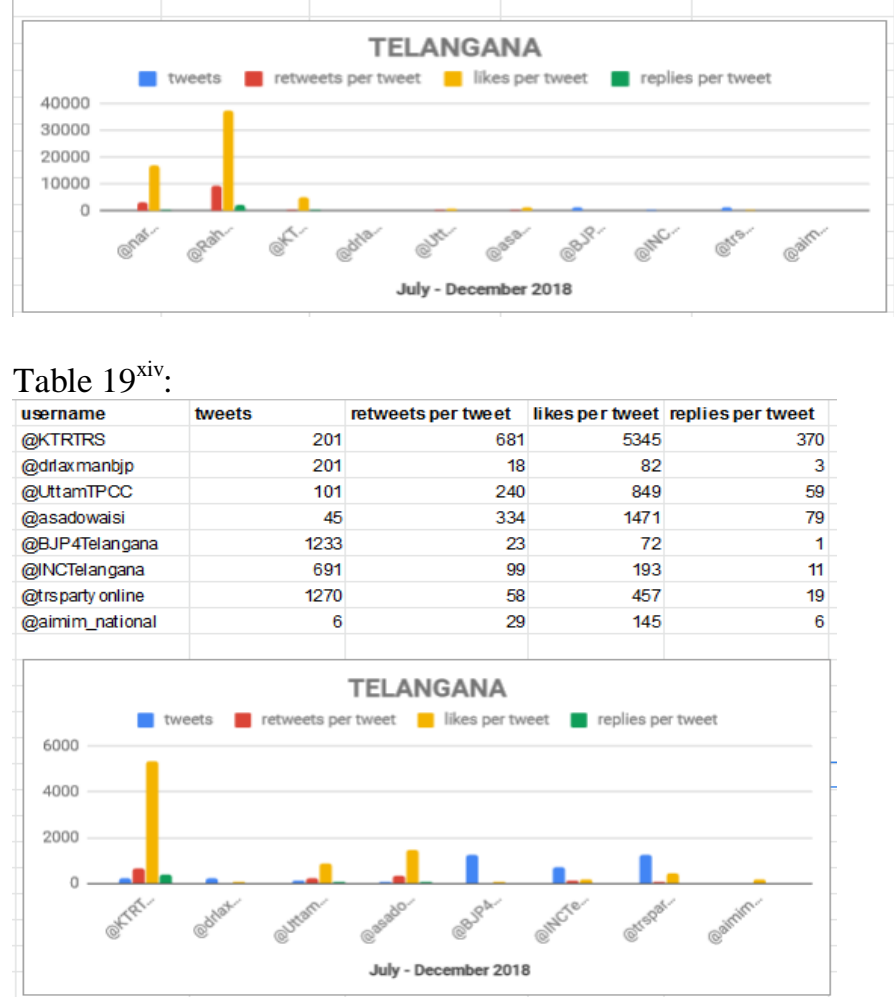

\section{KEY FINDINGS AND CONCLUSION}

The Congress had been in power for five years and faced strong opposition in the form of BJP in Karnataka and the Janata Dal (Secular) in south Karnataka during the polls in May 2018. BJP had announced former chief minister and Lingayat leader BS Yeddiyurappa as their chief ministerial candidate while the Janata Dal (Secular) was led by former chief minister and Vokkaliga leader HD Kumaraswamy. Narendra Modi and Rahul Gandhi remained the star campaigners for their parties in the elections. Rahul Gandhi had reworked his social media strategy and had started gaining major traction on Twitter right from the time of the 2017 Gujarat assembly elections. The trend continued in 2018 and the Karnataka assembly election was perhaps for the first time Rahul Gandhi was ahead of Narendra Modi in 
terms of average engagement on virtually all metrics. There is a correlation between Twitter engagement and the performance of political parties in the Karnataka assembly elections. Rahul Gandhi was getting far better engagement on Twitter than Narendra Modi at the national level while Siddaramaiah got the maximum traction among top state-level leaders. Similarly, Congress' vote share went up from $36.59 \%$ in 2013 to $38.04 \%$ in 2018 . BJP which had polled $19.89 \%$ in 2013 saw its vote share going up to $36.22 \%$ in 2018 .

But this had a different bearing on the election outcome which resulted in a hung assembly. Of a total of 224 seats in Karnataka, BJP witnessed a major rise from 40 seats in 2013 to 104 seats in 2018 while the ruling Congress was reduced from 120 seats in 2013 to 78 seats in 2018. The Janata Dal (Secular) saw a marginal dip in its fortunes from 40 seats in 2013 to 37 seats in 2018. BJP's rise was more to do with rebel leaders, BS Yeddiyurappa and B Sriramulu, returning to the party fold. Yeddiyurappa had formed his party, Karnataka Janata Paksha (KJP), and contested the 2013 elections garnering $9.79 \%$ vote share while B Sriramulu's BSR Congress Party (BSRCP) polled $2.69 \%$ vote share in the 2013 elections. There was a disconnect between Rahul Gandhi's Twitter handle and the party's main Twitter handle when it comes to raising certain issues during elections. Even though Rahul Gandhi was getting very high engagement, he put out very few tweets related to jobs (03) and corruption (04) compared to the main handle which posted 111 tweets on jobs and 80 tweets on corruption.

The 2018 Telangana assembly elections threw a very interesting trend on Twitter. While the top star campaigners of BJP and Congress - Narendra Modi and Rahul Gandhi were getting a lot of traction on Twitter, their state Twitter handles had very poor engagement levels in comparison. It was KT Rama Rao, a senior leader of the Telangana Rashtriya Samithi (TRS) and the son of Telangana chief minister K Chandrasekhar Rao who was getting a good response from Twitter users during the election campaign. KT Rama Rao was far ahead of the state-level competition on Twitter given by BJP state president Dr K Laxman and Congress state unit chief Uttam Kumar Reddy. The main Twitter handle of the ruling TRS and its leaders, especially KT Rama Rao relied heavily on the Telangana sentiment posting 1,270 and 201 related tweets respectively, but Rahul Gandhi did not adequately counter the TRS on this issue on Twitter posting only seven related tweets. BJP and Congress were too dependent on their national leadership and the lack of a credible local face gave the advantage to TRS. The Twitter engagement levels and the 2018 assembly election results have a clear correlation as TRS swept back to power with an improved performance. The number of seats in the 119 member Telangana assembly went up from 63 seats in 2014 to 88 seats in 2018 for TRS while Congress which was hoping to make a comeback had a marginal loss from 21 seats in 2014 to 19 seats in 2018. The TDP was decimated from 15 seats in 2014 to just two seats. AIMIM retained its seven seats from 2014 and BJP went down from five seats in 2014 to just one seat in the 2018 elections.
However, there is no clear causation between Twitter engagement and election results. Much more similar studies needed to be conducted before the same can be established.

\section{REFERENCES}

1. IANS, 'Narendra Modi as 'youth icon': BJP taps into social media to drum up support', 05.08.2013, India Today, https://www.indiatoday.in/india/north/story/narendra-modi-as-youth-ico n-bjp-taps-into-social-media-172828-2013-08-05

2. Sanjeev Ratna Singh, 'NARENDRA MODI VS RAHUL GANDHI ON TWITTER: A COMPARISON OF SOCIAL MEDIA STRATEGIES FOR POLITICAL MOBILISATION (2017-19)', Volume - 10, issue - 5 May 2019, International Research Journal of Management Sociology \& Humanities.

3. Ravi Sharma, 'Karanataka goes through a political upheaval as the Janata Dal (Secular) faction led by party supremo H.D. Deve Gowda's son H.D. Kumaraswamy withdraws support to the coalition government and partners with the Bharatiya Janata Party to form an alternative government.' Volume 23 - Issue 02, Jan. 28 - Feb. 10, 2006, The Frontline,

https://frontline.thehindu.com/static/html/fl2302/stories/200602100055 02100.htm

4. M Gautham Machaiah, 'Karnataka polls 2018: Kannadiga pride set to be pivotal factor in outcome', 31.03.2018, Moneycontrol.com https://www.moneycontrol.com/news/politics/karnataka-polls-2018-kan nadiga-pride-set-to-be-pivotal-factor-in-outcome-2540063.html

5. Yunus Y. Lasania, 'Telangana CM KCR dissolves assembly, to go for early elections', 06.09.2018, LiveMint, https://www.livemint.com/Politics/ve5G5jKFF0Hg4vAe4d43BM/Citin g-political-fragility-KCR-dissolves-Telangana-assembly.html

6. Ch. Sushil Rao, 'TRS gets social media troops battle-ready', 08.07.2018, The Times of India, https://timesofindia.indiatimes.com/city/hyderabad/trs-gets-social-media -troops-battle-ready/articleshow/64903953.cms

7. PTI, 'India has the cheapest mobile data in world: Study', 06.03.2019, The Economic Times, https://economictimes.indiatimes.com/tech/internet/india-has-the-cheape st-mobile-data-in-world-study/articleshow/68285820.cms?from $=\mathrm{mdr}$

8. Nandita Mathur, 'India's internet base crosses 500 million mark, driven by Rural India', 11.03.2019, Live Mint, https://www.livemint.com/industry/telecom/internet-users-exceed-500-m illion-rural-india-driving-growth-report-1552300847307.html

9. J. Clement, 'Leading countries based on number of Twitter users as of July 2019 (in millions)', 09.08.2019, Statista.com, https://www.statista.com/statistics/242606/number-of-active-twitter-user s-in-selected-countries/

10. PTI, 'Congress gets lesser seats in Karnataka though vote share nearly 2 percentage points higher than BJP', 15.05.2018, The Economic Times, https://economictimes.indiatimes.com/news/politics-and-nation/congress -gets-lesser-seats-in-karnataka-though-vote-share-nearly-2-percentage-p oints-higher-than-bjp/articleshow/64180347.cms?from $=\mathrm{mdr}$ 